\title{
El este y el sudeste asiáticos después de la crisis económica-política de 1997
}

$\mathrm{I}$ ntroducción

El objetivo del presente ensayo es presentar una lectura comparada de los diversos análisis acerca de la nueva realidad creada por la crisis económica iniciada el 2 de julio de 1997 en Asia del este y del sudeste. Por lo anterior, primero se examinará el impacto de dicha crisis a nivel regional, tanto en lo económico-financiero como social y político. Segundo, se presentarán las transformaciones más importantes que se realizaron en cada uno de los países más afectados por la crisis (Corea, Malasia, Filipinas, Indonesia y Tailandia).Se harán referencias al inicio de la crisis pero queda fuera del propósito del ensayo extenderse en el examen de sus causas, ya que en estos tres años ya existe una vasta bibliografía sobre el tema. ${ }^{1}$

La crisis económico-financiera, que se inició en Tailandia el 2 de julio de 1997 y que rápidamente se extendió al este y sudeste asiático cumplió en julio del 2000 tres años. Durante ese lapso, la economía y la sociedad de los países de esa región han experimentado profundos cambios en sus estructuras económicas, financieras, sociales y políticas.

Al referirse a esta crisis, Yilmaz Akyuz, jefe del Departamento de Macroeconomía y Desarrollo de la UNCTAD, afirma " En menos de un año, desde mediados de 1997 las economías del Este Asia pasaron de ser ejemplos de la experiencia de desarrollo más exitosa en la historia moderna al estancamiento y caída del crecimiento económico."2

El profesor Philip Kotler dice "Cuando la crisis financiera golpeó Asia en 1997 muchos creyeron que la región sería convertida en

* Embajador de México en Tailandia, Camboya, Laos y Vietnam de julio de 1995 a julio de 2001. ruinas. Lo que había sido la economía de más rápido crecimiento en el mundo se convirtió repentinamente en el peor lugar para invertir. Muchos analistas rápidamente encontraron culpables de la crisis; para los macroeconimistas la caída de Asia se debió a las políticas macroeconómicas. Los analistas financieros y bancarios vieron la culpa en la debilidad del sistema bancario asiático. La causa de la crisis asiática pudo ser discutida, pero sus resultados no: el milagro asiático parecía haberse terminado." 3

\section{La crisis, consecuencia de una lectura equivocada del mercado}

La crisis iniciada en julio de 1997, y su rápida expansión por el resto de los países del sudeste de Asia y luego a Corea y Hong Kong, fue casi unánimemente comparada, tanto por los líderes políticos, empresariales e inclusive algunos académicos de los países de esas regiones, con un terremoto, con lo que querían decir que su aparición fue algo similar a un fenómeno natural, que es difícil predecir con certitud. Otra explicación, que estuvo de moda en los primeros meses, fue que la misma era resultado de la acción de fuerzas extrarregionales, y no faltó quien las identificara con los fondos de inversión que operan en el mercado de valores. $\mathrm{Al}$ respecto, se dieron como ejemplo las operaciones realizadas por personajes como Georges Soros, el autor de The crisis of global capitalism y dueño del Quantum Fund, mismo que por cierto ya no existe.

$\mathrm{Al}$ promover esos enfoques de la crisis, las élites políticas, empresariales y sus partidarios en otros sectores sociales pretendían evadir su responsabilidad por no haber interpretado correctamente y a tiempo las señales que desde 1995 les estaba mandando el mercado. Después de tres años de crisis, esa actitud está cam- 
biando. Un ejemplo es lo dicho por el ex primer ministro de Tailandia, Anand Panyarachun, una de las personalidades que goza de reconocido prestigio en el país :

"La situación en Asia en 1996 y 97 demuestra (un) retraso en entender las señales de alarma que daba la información. Debimos de haber observado el descenso en el mercado de valores, la caída en los precios de los bienes raíces, en las exportaciones, los déficit en la cuenta corriente y las presiones sobre nuestra moneda como un aviso de que marchábamos por el camino equivocado. Me temo que estábamos muy seguros de que íbamos por la vía correcta, y tan orgullosos del alto ritmo de crecimiento de nuestra economía que no estábamos preparados para escuchar la alarma... Ahora debemos limpiar los escombros de la crisis financiera, asegurarnos que hemos tomado la dirección correcta, ( si necesario) cambiarla o disminuir el ritmo.." 4

El estadista Lee Kuan Yew, ex primer ministro de Singapur y respetada personalidad en la región, en una conferencia que dictó en el Colegio de la Defensa Nacional de Tailandia, el 21 de junio del 2000, afirmó: "la mayoría de los préstamos- obtenidos en los años 80 cuando Asia del Este era la preferida de los inversionistasse invirtieron en el sector de bienes raíces y en el mercado de valores, lo que creó la "burbuja" económica que explotó... por culpa de los gobiernos que no fueron capaces de controlar el excesivo endeudamiento, particularmente de los empresarios, más aún por que ellos incitaron ese proceso al adoptar políticas que crearon un ambiente de euforia." ${ }^{5}$

Otro destacado líder singapurense, el viceprimer ministro, Lee Hsien Loong, al referirse a los diferentes enfoques de los economistas tanto del Fondo Monetario
Internacional como de otros organismos, apunta "visto en retrospectiva se equivocaron respecto a la magnitud del problema, sobre su impacto y así como con respecto a la reacción de los inversionistas que fue una rápida reacción global de retirada de todas las posiciones." 6

El mismo funcionario singapurense considera que "La crisis que golpeó a Asia en 1997 mostró lo inestable que puede ser el sistema financiero internacional, lo vulnerable que eran las aparentemente fuertes economías de Asia a un choque inesperado. Ello originó que se reexaminaran los beneficios y riesgos de la globalización así como los lados fuertes y débiles de las economías asiáticas."7

Para los expertos de la Comisión Económica y Social para el Asia y el Pacífico (ESCAP), la crisis en Asia tuvo tres causas principales: una dramática caída en el crecimiento de las exportaciones, las políticas de liberalización de los mercados de capital y un "duro" comportamiento de los mercados extranjeros. ${ }^{8}$

Así, el primer cambio fue que en los diferentes países del este y sudeste de Asia se aceptaron dos componentes importantes del origen de la crisis iniciada en 1997: uno, la falta de visión de los líderes políticos para entender las señales del mercado para hacer los cambios en las estructuras financiera, bancaria, industrial y política que permitiera a sus economías adaptarse a las transformaciones experimentadas en la economía global y, otro, la complacencia de los inversionistas extranjeros y de los administradores de portafolios en las bolsas de valores.

Para entender la teoría sobre la crisis asiática del profesor Kotler hay que comprender la manera asiática del desarrollo económico que comprende los siguientes elementos: gobierno 
autoritario, economía estatal, instituciones, valores asiáticos y las redes (network). ${ }^{9}$

\section{Consecuencias sociales de la crisis}

La crisis provocó, en primer término, una severa caída de la tasa de crecimiento de las economías de los países de Asia del este y del sudeste y un fuerte impacto en el empleo. Las tasas anuales (1998) de desempleo fueron impresionantes: en Malasia y Tailandia de 130 por ciento de alrededor de 200 por ciento en Corea y de más de 350 por ciento en Indonesia. En términos de desempleados (1998) en Malasia unos 600,000, en Corea y Tailandia 1.5 millones y unos 20 millones en Indonesia ${ }^{10}$

Las mujeres, que durante el auge económico representaron un importante porcentaje en el mercado de mano obra, fueron uno de los sectores más golpeados dado que las empresas que las empleaban, como la textil, la de la construcción, las de manufacturas, los bancos, fueron las más afectadas por la crisis. En Tailandia, las mujeres, que constituían 80 por ciento de trabajadores no calificados en el sector industrial, fueron despedidas; en Corea, donde representaban 60 por ciento de los empleados en negocios con menos de 5 personas, se quedaron sin trabajo cuando pequeñas empresas quebraron.

A este problema se agrega el de la repatriación de trabajadores a sus países de origen. De Tailandia salieron unos 250,000, principalmente hacia los países vecinos; unos 50,000 dejaron Malasia. A su vez a Tailandia regresaron buen número de tailandeses desde Corea, Taiwan, Malasia o Singapur.

Se deterioró el monto de los salarios, cayó el ahorro y el poder de compra, por los recortes en los presupuestos bajó la calidad de los servicios de salud y de educación. Lo anterior se expresó en un evidente incremento de pobreza, índice de criminalidad, violencia intrafamiliar, número de suicidios, prostitución, tráfico de drogas y descontento político. Como respuesta, se adoptaron diversos programas urgentes de asistencia para hacer frente al impacto social de la crisis. ${ }^{12}$

En Indonesia se inició un programa, con un total de 1,800 millones de dólares, orientado básicamente a los habitantes de poblaciones rurales para garantizarles abasto de productos a precios accesibles, y promover proyectos con empleo intensivo de mano de obra. La Organización Mundial de la Salud apoyó programas de compra de medicamentos de los países de la región haciendo ahorros importantes al no importarlos de fuera de la zona. Malasia puso en práctica mecanismos para evitar aumentos excesivos de precios en productos como aceite para cocinar, arroz, azúcar, etc. Corea puso en operación un programa de seguro del desempleo, financiamientos subsidiados a los desempleados, y Tailandia emprendió un programa titulado "proyecto de inversión social" con un financiamiento de 462 millones de dólares incluyendo apoyo a la creación de empleos, reentrenamiento para trabajadores, acceso a los servicios de salud y el desarrollo de proyectos

Cuadro 1

\begin{tabular}{|c|c|c|c|}
\hline & 1997 & 1998 & \\
\hline & $\begin{array}{l}\text { Tasa de desempleo } \\
\text { (porcentaje) }\end{array}$ & $\begin{array}{c}\text { Tasa de desempleo } \\
\text { (porcentaje) }\end{array}$ & $\begin{array}{c}\text { Número de desempleados } \\
\text { (millones) }\end{array}$ \\
\hline Indonesia & 4.7 & $21.3 \mathrm{a}$ & 20 \\
\hline Malasia & $2.7 \mathrm{~b}$ & $6.4 \mathrm{c}$ & 0.6 \\
\hline Corea & 2.6 & $7.7 \mathrm{c}$ & 1.5 \\
\hline Tailandia & 1.9 & 4.4 & 1.5 \\
\hline
\end{tabular}


comunitarios, tanto en las zonas rurales como urbanas.

\section{Repercusión en los bienes raíces}

Los bienes raíces, que en varios países captaron considerables inversiones, fueron unos de los primeros en sufrir el impacto de la crisis, pues obligó a la mayoría de los inversionistas del sector a abandonar proyectos, dejando a quienes tenían contratos de compra con estructuras iniciadas o semiterminadas o con porciones de tierra y sobre los cuales ni siquiera los derechos de propiedad podían reclamar.

Un buen ejemplo de lo anterior es Hong Kong, ciudad preferida como sede por muchas compañías trasnacionales, donde los precios de los bienes raíces cayeron más de 50 por ciento desde mediados de 1997, el número de transacciones también disminuyó drásticamente: en el 2000 solo se registraron 85,744 operaciones 58 por ciento comparadas con las 205,461 de 1997 . El valor de las operaciones en el 2000 también bajó un 75 por ciento equivalente a Hong Kong dólares $222.5 \mathrm{mil}$ millones ( $\$ 28.5$ mil millones de dólares) con respecto a HK $\$ 868$ mil millones en 1997 . Tanto el número de operaciones como su monto fue el menor de los últimos diez años ${ }^{13}$.

Al respecto, el profesor y conocido periodista Walden Bello afirma que en Manila los analistas de mercado proyectaban que para el 2000 la oferta de condominios residenciales excedería la demanda en más de 211 por ciento y por lo que se refiere a los espacios para comercios, sobrepasaría la demanda en un 142 por ciento. ${ }^{14}$; Existe una sobreoferta de bienes raíces en Kuala Lumpur y Jakarta, ya que los préstamos de los bancos comerciales a dicho sector representaron un alto porcentaje del total de su cartera vencida: en Filipinas entre el 15 25 por ciento, el 20-25 por ciento en Malasia, y 40 por ciento en Tailandia, según un reporte del diario The Nation (13 de noviembre del 2000) En el caso de este país, la crisis sacó del mercado de bienes raíces a más de 1,000 empresas.

\section{¿Se inició la recuperación?}

Ya en 1999 tanto en el este como en el sudeste asiáticos se planteó la pregunta ¿ los países de la región entrarían en el nuevo milenio administrando la crisis o ya habrían dejado atrás esa pesadilla? Al respecto es ilustrativo reproducir algunos de los encabezados de la prensa y comentarios de revistas en aquellos días:

"Asia is rebounding, but not that quickly", The Nation, 22 de diciembre de 1999; " The risk takers, amid rubles, savy entrepreneurs are layin theirs bets on recovery", Newsweek, november 2 de 1998; "Recovery won't last without real reforms, The Nation,12 de enero del 2000; "East Asia's revival seems to be assured, but shaded by risks", International

Cuadro 2

\begin{tabular}{lrrrrrr}
\hline & PIB & \multicolumn{7}{c}{ INFLACIÓN } & 2000b/ \\
\hline & 1998 & $1999 \mathrm{a} /$ & $2000 \mathrm{~b} /$ & 1998 & $1999 \mathrm{a} /$ & 5.4 \\
Sudeste de Asia & -6.9 & 3.4 & 4.5 & 27.6 & 9.3 & 4 \\
Indonesia & -13.7 & 0.1 & 3 & 77.6 & 20.4 & 3 \\
Malasia & -7.5 & 5.4 & 5.8 & 5.3 & 3 & 7 \\
Filipinas & -0.5 & 3.2 & 4.5 & 9 & 7 & 1 \\
Singapur & 0.4 & 5.4 & 6 & -0.3 & 0.4 & 2.5 \\
Tailandia & -10.4 & 4.1 & 4.4 & 8.1 & 0.3 & 1.5 \\
Noreste de Asia & 1.6 & 6.7 & 6.9 & 2.7 & -0.4 & -3 \\
Hong Kong & -5.1 & 0.5 & 4 & 2.8 & 0.9 & 3.2 \\
Corea & -5.8 & 9 & 7.8 & 7.5 & & 0.3
\end{tabular}

Fuente: Economic and Social Survey of Asia and the Pacific 2000. Economic and Social

Commision for Asia and the Pacific, United Nations 2000, p. 24

a: estimado

b: proyección 
Herald Tribune, 8-9 october 2000; “A false dawn. Changing economic conditions may mean that region's much-touted recovery from crisis is largely an illusion", Far Eastern Economic Review, july 27, 2000; "Asia "recovery" may be running out of steam", The Nation, 19 de octubre del 2000; "Timing the turnaround. Fund managers are divided over whether Asian markets are nearing bottom as they focus on individual companies and when to get back in", Far Eastern Economic Review, 26 de octubre del 2000; "Asia stocks tumble on oil costs", International Herald Tribune, 14-15 de octubre del 2000; " Need for economic reform in Asia grows", The Nation,3 de noviembre del 2000.

A pesar de los puntos de vista divergentes en cuanto al ritmo de la recuperación en Asia del este y del sudeste y respecto a si la misma es sostenible, la evaluación de organismos internacionales como (ESCAP), en su informe anual editado en el 2000, es la que se aprecia en el cuadro 2.

$\mathrm{Al}$ respecto, especialistas como el profesor Paul Krugman en su artículo "The return of depression economics"15 también fue de la idea de que en 1999 " habría una módica oportunidad de que observaramos cierta recuperación económica en Asia..”

Los análisis de la crisis asiática en términos generales coinciden en que en 1999 las economías del este y sudeste Asiático -con la excepción de Indonesia-reinician el crecimiento económico. Sin embargo, durante el primer semestre del 2000 persistió la duda acerca de si la recuperación que se inició durante 1999 y se mantiene en el curso de los primeros meses de este año es sólida, o si va a derrumbarse bajo el peso de las crecientes ineficiencias, por el aumento de la deuda pública, ante la nueva competencia en el mercado mundial o ante el incumplimiento de la promesa de reestructurar la banca y las empresas ${ }^{16}$. En el mismo contexto se pregunta ¿ puede convertirse la recuperación en una era de alto crecimiento? La respuesta difiere.

Para los expertos del Banco Mundial "La herencia de la crisis, en especial el fuerte endeudamiento y la gran inseguridad para los trabajadores, hacen que la recuperación esté expuesta a cambios inesperados de la actitud de los inversionistas"17.

En el Foro organizado por el Instituto de Estudios del Sudeste Asiático "Asia del este, de la crisis a la oportunidad" si bien se reconoció que la recuperación ha sido más rápida de lo esperado, también se observó que es "desigual”, ya que por ejemplo Corea obtuvo 10.2 por ciento de crecimiento en 1999 y en cambio Indonesia sigue rezagada debido a problemas internos; igualmente dicha recuperación no ha sido generalizada, pues aun cuando las bolsas de valores muestran recuperación, el mercado de bienes raíces sigue con problemas y la inversión privada y el consumo interno aún deben recuperarse. Por ello, se advirtió que para evitar otra crisis la región debe resistir la tentación de disminuir el ritmo de las reformas, a pesar de lo dolorosas que sean. ${ }^{18}$

La Organización para la Cooperación y el Desarrollo Económico(OCDE) en su reporte de mediados del 2000, dice que " aun cuando los riesgos de otra caída han disminuido

Cuadro 3

Los pronósticos de la OCDE para la región son positivos

\begin{tabular}{|c|c|c|c|c|c|c|}
\hline & $\begin{array}{l}\text { Crecimiento } \\
\text { del PIB en \% }\end{array}$ & \multicolumn{4}{|c|}{ Inflación en \% } & \\
\hline & 1999 & 2000 & 2001 & 1999 & 2000 & 2001 \\
\hline Hong Kong & 2.9 & 5.2 & 5.5 & -3.3 & -0.5 & 1.5 \\
\hline Indonesia & -0.5 & 3 & 4.2 & 20 & 2.6 & 5 \\
\hline Malasia & 5.1 & 6.2 & 6 & 0 & 2.5 & 3.5 \\
\hline Filipinas & 3 & 3.5 & 3.7 & 7 & 5 & 5 \\
\hline Tailandia & 4.2 & 5.5 & 6.5 & 0 & 2 & 3.5 \\
\hline
\end{tabular}

Fuente: OCDE, gráfica del diario Bangkok Post , 31 de mayo de 2000. 
considerablemente, no han desaparecido del todo" y que el mayor peligro externo para los países de la región es "el aterrizaje de la economía de los Estados Unidos en especial si el mismo se ve acompañado de un fuerte incremento de las tasas de interés." A lo cual hay que agregar el comportamiento errático de la economía japonesa.

La advertencia de la OCDE sobre las consecuencias de la recuperación económica en Asia del este y del sudoeste, dependiendo del comportamiento de la economía estadounidense, es de alguna manera apoyada por Alan Greenspan, presidente de la Reserva Federal de los EUA, al afirmar que una nueva crisis financiera es inevitable "Nosotros no sabemos y probablemente no podremos saber la naturaleza precisa de la próxima crisis financiera.. . es certero que la habrá, dada la persistencia de la indiscreción humana y financiera" ${ }^{19}$

En el "Panorama económico para la región de Asia en proceso de industrialización en el 2000" del Instituto para Economías en Desarrollo (IDE) de Japón, también se afirma que en 1999 las economías de la región se orientan hacia la recuperación, ya que está previsto que todos los diez países (del sudeste de Asia) y las regiones del este de Asia obtendrán tasas positivas de crecimiento, aunque de diferente nivel. ${ }^{20}$

Los análisis citados reconocen, por un lado, que la crisis en términos generales ya tocó fondo y que la región, aunque a ritmos diferentes, inició su crecimiento y, por otro, lado que persisten tres preocupaciones centrales acerca de dicha recuperación: una, tiene que ver con el rezago en el sector de bienes raíces, otra, con los préstamos no recuperables en la banca y, la tercera, con la percepción de que se pierde interés en llevar adelante las reformas y la reestructuración de los sectores financiero e industrial.

\section{Sector de bienes raíces}

El paisaje urbano en las principales ciudades del este y sudeste de Asia confirma que en el sector de bienes raíces es donde la recuperación avanza más lentamente, debido a que no regresa a la velocidad deseada la confianza de los inversionistas.

Ese panorama desalentador se modificó al final del 2000; así lo confirma en su último informe la compañía consultora en bienes raíces Jones Lang Lasalle al afirmar que después de aproximadamente tres años de la caída de los precios y de permanecer semivacíos los rascacielos, el mercado de bienes raíces está en el camino de su recuperación. ${ }^{21}$

Según dicha consultoría, a fines del 2000 se registró un notable incremento en la demanda de espacios para oficina en Pekín Shanghai, Hong Kong,Tokio, Singapur. La compañía Morgan Stanley Asia Ltd, esperaba que los precios de la propiedad residencial en Hong Kong se incrementarán entre $10-15$ por ciento en el 2001, pero este pronóstico optimista no lo comparten todos los empresarios del sector porque tienen presente la importante oferta de departamentos de la empresa oficial Home Ownership Scheme(HOS). ${ }^{22}$

Es importante destacar que dicha recuperación también se está dando en forma desigual, más rápida en los países de Asia del norte que en los del sudeste. En Bangkok, las múltiples construcciones que siguen esperando las inversiones que permitan terminarlas, los condominios que fueron concluidos pero que no tienen compradores, así como la abundante oferta de espacios para oficina o residencia, son claros ejemplos.

\section{Sector bancario}

En cuanto al sector bancario, de acuerdo con la compañía de valores DBS Group Holdings la buena noticia es que los bancos en Asia empiezan a resurgir de la crisis más sanos y con un criterio de mayor responsabilidad frente a los cambios inevitables que implica la competencia global, los rápidos movimientos de los flujos de capital, el comercio electrónico, la revolución en las comunicaciones y la tecnología de punta; sin embargo, el mayor peligro para muchos bancos asiáticos - quizás mayor ahora que durante la 
crisis- radica en una falsa percepción de seguridad. Adormecidos, como estuvieron en otros tiempos, por los vientos favorables y seducidos por las tentaciones de retornar a las viejas prácticas, muchos bancos asiáticos no aprovecharon las ventajas que les ofreció el clima favorable del año pasado (1999), al resistirse a la reestructuración, a las reformas y a redefinir sus estrategias. Así, se les agota el tiempo, lo reconozcan o no. ${ }^{23}$

"De regreso en los negocios, después de haber sido golpeados por la crisis, los 500 bancos más grandes de Asia vencen las dificultades. Ahora enfrentan los retos de su consolidación”. Así titula la revista Asiaweek un extenso análisis sobre la situación de la banca en el este y sudeste $\mathrm{Asia}^{24}$. El comentario apunta que parece que la costra de moho que cubría las estructuras de los bancos asiáticos finalmente ha sido removida. Algunas manchas aún son difíciles de quitar como en el caso de Indonesia, pero en muchos otros países las reglas del mercado han sido fortalecidas, los bancos han sido reestructurados, recapitalizados y los préstamos no recuperables removidos. ${ }^{25}$ El reto de hoy día es mantener su rentabilidad y cumplir con las exigencias de la globalización y de la nueva tecnología.

El Banco Asiático del Desarrollo(ADB) en su "Asia Recovery Report 2000" afirma que "La recuperación de Asia de la crisis financiera de 1997 ha resultado ser más rápida de lo que se esperaba. Las economías de los países más afectados -Indonesia, Corea, Malasia, Filipinas y Tailandia- han iniciado el despegue en la segunda mitad de 1998. La recuperación, como tal, comenzó a principios de 1999 con el reinicio del crecimiento, en un principio como resultado de las políticas de ajuste fiscal y monetario, luego por un ambiente económico global favorable. En general las exportaciones fueron el motor de la reactivación económica en un contexto de una demanda interna deprimida.." 26

\section{La recuperación de los efectos sociales de la crisis}

Por lo que se refiere a los avances en la tarea de superar las consecuencias sociales de la crisis,
ESCAP, en su Informe Económico y Social de Asia y el Pacífico en el 2000, constata que el impacto social de la crisis económica fue severo y generalizado y sus consecuencias aún están por verse. Los gobiernos han aplicado varios programas orientados a mitigar los efectos más graves de la crisis, como el desempleo, pero lo cierto es que aún amplios sectores de la población, particularmente aquellos que no están organizados tanto en zonas urbanas como rurales, siguen prácticamente excluidos de la cobertura de seguridad social.

Para el ADB la recuperación económica registrada en el 2000 trajo como consecuencia un mejoramiento del empleo, un incremento real en los salarios y en el consumo privado. En Indonesia, Corea y Tailandia, las tasas cuatrimestrales de desempleo en el 2000 fueron más bajas que las registradas en el mismo período de 1999, solo en Filipinas aumentó el desempleo, a pesar de su crecimiento económico. ${ }^{27} \mathrm{La}$ crisis reveló la urgencia de mejorar los mecanismos existentes de seguridad social: sus modalidades financieras, el tipo de servicios y la supervisión de su manejo.

\section{La crisis y su impacto en el sector financiero}

La crisis demostró que las economías del este y sudeste de Asia son más interdependientes y que la responsabilidad de un país en el crecimiento y desarrollo de otros es cada día mayor.

También promovió un interés mundial en la reforma del sistema financiero internacional, sobre todo la que tuviera como objetivos una mayor cooperación de la comunidad internacional para intercambiar información sobre asuntos económico-financieros con el fin de evitar las consecuencias negativas del contagio, como las experimentadas por las crisis en México, en Asia, Rusia o Brasil; para ello se aprobó poner en práctica un sistema de alerta temprana que permitiera interpretar correctamente las señales del mercado y prevenir crisis, clasificadas por el Fondo Monetario Interncional en monetarias, bancarias y de deuda externa. 
A nivel regional, los países miembros de la ANSEA, establecieron en su reunión de Manila, en noviembre de 1997, "un grupo (framework group ) para fortalecer la cooperación regional y promover la estabilidad financiera" y sobre la base de las recomendaciones de ese grupo, en 1998, los ministros de finanzas de la Asean decidieron establecer un sistema de monitoreo, mediante el cual el "grupo de trabajo de Manila" se reuniría dos veces al año para discutir, informalmente, la situación económica regional, el progreso en las reformas y la recuperación económica en general.

Un paso más en el fortalecimiento de esa cooperación fue la iniciativa de Chiang-Mai, mediante la cual se creó un mecanismo de cooperación regional para apoyar las monedas en momentos de crisis (ASEAN Swap arrangement*ASA*) que comprende, además de los 10 países de la Asean, China, Corea y Japón, o ANSEA+3. Algunos analistas apuntan que ciertos países asiáticos desearían ver evolucionar ese mecanismo hacia un Fondo Monetario Asiático que apoyaría al Fondo Monetario Internacional.

Los países que crearon el ASA acordaron dotarlo de un fondo de 1 billón de dólares. La base del ASA son acuerdos bilaterales por tres o seis meses entre los países miembros, conforme con los cuales los participantes pueden utilizar recursos para financiar su balanza de pagos en tiempo de crisis.

En esa tarea el Banco Asiático para el Desarrollo ofrece el apoyo técnico necesario. ${ }^{28}$

\section{Perspectivas para el 2001}

La Comisión Económica y Social de la ONU para Asia Pacífico (ESCAP, por sus siglas en inglés) en su Informe Económico y Social 2001 afirma que en términos de crecimiento las subregiones de sudeste y este de Asia fueron las que mostraron ( en el 2000) los mejores resultados ya que no sólo superaron el crecimiento del PIB de 1999 en 2 por ciento sino que además excedió significativamente el crecimiento previsto en los 12 meses precedentes (E, ESCAP, 1198,1 de marzo del 2001)
De acuerdo con ESCAP, en su informe citado en el párrafo anterior, las tasas de crecimiento y de inflación para la región en el período 1999-2003 serán las que se aprecian en el cuadro 4.

Pero ESCAP también advierte que las perspectivas a corto plazo para la región son mixtas, en razón tanto de su vulnerabilidad ante los riesgos externos (baja del crecimiento en la economía de Estados Unidos, marasmo económico y carencia de liderazgo en Japón, altos precios del petróleo), como por problemas domésticos: incremento de la deuda pública, debilidad en la tasa de cambio, baja en el mercadobursátil.

Los principales desafíos, según el informe citado de ESCAP son, por un lado, para la región en su conjunto, mantener el ímpetu del crecimiento en el 2001 ante los factores externos desfavorables y, por otro en el ámbito nacional, que todos los gobiernos se comprometan a mantener un balance macro económico, a consultarse con mayor frecuencia en el ámbito regional sobre cuestiones políticas (por ejemplo sobre el avance en el cumplimiento de los acuerdos sobre liberalización comercial), o sobre la necesidad de evitar la toma de decisiones nacionales sobre problemas comerciales y financieros que puedan tener efectos desestabilizadores en otros países.

Otro problema común ligado a lo anterior es la percepción de que en muchos países de la zona persiste un lento progreso en la estructuración de los sectores financiero y corporativo. Esa impresión de fatiga de las reformas debe ser superada acelerando dichos procesos fijándose metas precisas, fortaleciendo las instituciones y los procedimientos correspondientes, como los de las agencias encargadas de recuperar las deudas o las cortes encargadas de los asuntos de quiebras. ${ }^{29}$

Respecto a las perspectivas económicas para 2001 el ADB es más preciso que el Informe de ESCAP al afirmar que "La recuperación de los cinco países más afectados por la crisis (Corea, Malasia, Filipinas, Indonesia y 
Tailandia), debido al impacto en la región de los riesgos externos apuntados en párrafos anteriores en el 2001, disminuirá su crecimiento a 4 por ciento en promedio, comparado con 7.1 por ciento obtenido en el 2000 , pero estima que dicho crecimiento alcanzará 5 por ciento en el 2002.

Las exportaciones que fueron el motor de la recuperación empezaron a disminuir a partir de septiembre-octubre del 2000 y se espera una mayor caída en el 2001, en cambio las baja en la tasa de interés en los Estados Unidos puede tentar a los inversionistas a regresar a los mercados regionales de acciones; por primera vez, desde la crisis, se prevén flujos de capital privado.

Si bien, en el ámbito doméstico, los riesgos políticos han disminuido en Filipinas y Tailandia, aún la confianza de los inversionistas no se ha completamente recobrado.

Se espera que el impacto adverso de la baja en la demanda en el mercado mundial y en la industria de la electrónica será significativo.

Los temores de una nueva crisis han sido exagerados, ya que la región está en mejores condiciones de absorber el impacto de los riesgos

\begin{tabular}{|c|c|c|c|c|c|}
\hline \multicolumn{6}{|c|}{$\begin{array}{c}\text { Cuadro } 4 \\
\text { PIB real } \\
\end{array}$} \\
\hline \multicolumn{2}{|c|}{$19992000 \mathrm{~b} /$} & $2001 \mathrm{c} /$ & $2002 c /$ & \multicolumn{2}{|l|}{2003} \\
\hline Sudeste Asia & 3.4 & 5.9 & 5.2 & 5.5 & 5.9 \\
\hline Indonesia & 0.2 & 4.4 & 5 & 5.7 & 6.1 \\
\hline Laos,Rep.Dem. & 7.3 & 6.5 & 6.9 & 7.2 & 7.3 \\
\hline Malasia & 5.4 & 8.2 & 7 & 7.2 & 7.5 \\
\hline Filipinas & 3.3 & 3.7 & 3.4 & 3.8 & 4.6 \\
\hline Singapur & 5.4 & 10.1 & 6.3 & 6.3 & 6.2 \\
\hline Tailandia & 4.2 & 4.5 & 4.6 & 4.6 & 5.2 \\
\hline Vietnam & 4.8 & 6.7 & 7 & 7 & 7.5 \\
\hline Norte/este Asia & 7.6 & 8.2 & 6.4 & 6.5 & 6.8 \\
\hline Hong Kong,China & 3 & 9.7 & 6.1 & 5.2 & 5.2 \\
\hline Corea & 10.7 & 9.2 & 5.1 & 5.8 & 5.9 \\
\hline \multicolumn{6}{|l|}{ Inflación a/ } \\
\hline sudeste Asia & 7.3 & 2.5 & 4.2 & 4.6 & 5.1 \\
\hline Indonesia & 20.5 & 3.7 & 7 & 7.6 & 8.8 \\
\hline Laos Rep.Dem. & 134 & 15 & 10 & 10 & 10 \\
\hline Malasia & 2.8 & 1.8 & 2.9 & 3.2 & 3.8 \\
\hline Filipinas & 6.7 & 4.3 & 6.1 & 5.6 & 5.3 \\
\hline Singapur & 0.1 & 1.5 & 2.1 & 2 & 2.3 \\
\hline Tailandia & 0.3 & 1.7 & 2.6 & 3.5 & 3.7 \\
\hline Vietnam & 4.3 & -1.5 & 4.5 & 6.5 & 7 \\
\hline Norte/este Asia & -0.6 & 0.9 & 2.4 & 3.4 & 4 \\
\hline Hong Kong, China & -4 & -3.2 & 2.1 & 3.7 & 4.2 \\
\hline Corea & 0.8 & 2.3 & 3.4 & 3.2 & 3.6 \\
\hline \multicolumn{6}{|c|}{$\begin{array}{l}\text { International Financial Statistics.vol. LIII, núm.9 (september 2000); "Asian Development Bank, } \\
\text { Key Indicatos of Developing Asian and Pacific Countries" (Oxford Universsity Press, 2000) } \\
\text { United Nations, "Project LINK World Economic Outlook"(october 2000). } \\
\text { b: estimado }\end{array}$} \\
\hline
\end{tabular}


externos en razón de que: la calidad de la recuperación está mejorando, se reduce la vulnerabilidad del sector financiero, se fortalece la capacidad externa de pago y hay una mayor coherencia en las políticas macroeconómicas.

La crisis generó impactos negativos y positivos en cuanto a la inversión extranjera directa. El lado negativo fue que la caída de la demanda interna y la perspectiva de la disminución en el crecimiento desalentó la inversión extranjera al mercado interno, lo cual se agravó con la incertidumbre política del inicio de las medidas de ajuste."

Del lado positivo, la depreciación de las monedas redujo los costos internos de producción, así como el valor de las acciones de las empresas, lo cual hizo rentable la inversión extranjera y, además, la revisión de la legislación sobre dicha inversión, que formó parte de los paquetes de reestructuración que abrió nuevas opciones para las operaciones de fusión y adquisición de compañías.

Durante el período anterior a la crisis, la inversión extranjera aumentó considerablemente de 1.7 mil millones en 1980-1984 a 20 mil millones en 1996. En 1998, cuando la crisis llegó a su clímax, el flujo de la inversión extranjera cayó de 19.2 mil millones en 1997 a 16.7 mil millones en 1998, para luego aumentar a 17.4 mil millones en 1999. Además agrega el ADB:

"La fusión y adquisición de compañías ha sido el eje de la reestructuración de los bancos y de las empresas en los países afectados, lo que ha contribuido a una recuperación sostenida y también al incremento de los flujos de inversión extranjera."

\section{Después de la crisis: una Asia del norte rica y una Asia del sur pobre}

Se aprecia que hay un consenso en el sentido de que el este y sudeste de Asia han iniciado su recuperación de la crisis, pero también la advertencia de varios analistas tanto de que dicha crisis contribuyó a profundizar la diferencia entre Asia del norte y del sur.

Tom Holland escribe en Far Eastern Economic Review que " la línea que pasa 20 grados norte de la frontera de Tailandia y sigue al oriente entre Filipinas y Taiwan marca la división entre dos zonas económicas que rápidamente se van diferenciando. Al norte las economías están prosperando (are booming), al sur el progreso se percibe más bien inestable. En el norte la disminución del riesgo político, importantes ingresos de inversiones y la tendencia hacia las nuevas tecnologías están ayudando a forjar una robusta economía del futuro. En contraste, el crecimiento en el sur se ve inestable. La inestabilidad política ha entrampado la reforma en Indonesia y en Filipinas. En Tailandia una reestructuración tuortosa ha hecho que se pierda la fe en un país que muchos creyeron guiaría al Asia para salir de la crisis. En Malasia el crecimiento parece fuerte, pero la política gubernamental de tipo de cambio augura problemas a futuro. \& Y Singapur? Él sufre de ser una casa en orden en un mal (lousy) vecindario" 30

Similar punto de vista expresó Michael Richardson: "El resurgimiento de Asia del este de la crisis financiera de 1997 y 1998 ha superado las expectativas. Pero algunos funcionarios y banqueros están preocupados por que la rapidez de la recuperación alimenta la complacencia y quizás siembra las semillas de una nueva crisis" ${ }^{\prime 1}$

Idéntica preocupación expresa The Economist al afirmar que el estancamiento de las reformas en el caos político continúa frenando a Tailandia, Indonesia y Filipinas. ${ }^{32}$

Ejemplos de lo anterior es la resistencia, calificada por algunos comentaristas como "nacionalista", de ciertos grupos empresariales fuertemente endeudados para impedir que la corte les imponga planes de reestructuración, como por ejemplo la lucha que libró Prachai Leophairatana, principal accionista de una de las grandes compañías de Tailandia, Thai Petrochimical Industry(TPI), Kim Woo Choong 
y la familia Chung Ju-yung, respecto a los grupos coreanos Daewoo y Hundai.

Lo anterior confirma que no todos en la región han entendido que las reglas para los negocios están cambiando rápidamente, que los elementos que antes favorecieron el crecimiento de la región, que algunos califican de " modelo asiático", acerca del cual siempre hubo una definición nebulosa, ya no existen después del fin de la Guerra Fría. Por ejemplo: hoy la prioridad es la relación comercial y no la seguridad; los flujos de inversión los dominan las compañías privadas; las operaciones en las bolsas de valores y los créditos y prestamos son prioritariamente para apoyar la productividad y competitivad empresarial y no para garantizar la seguridad nacional. ${ }^{33}$

En otros miembros del "trío problemático" como lo calificó The Economist, la situación sigue siendo muy preocupante: en Indonesia las demandas separatistas, la resistencia a los intentos del gobierno de llevar a la justicia al ex presidente Suharto y a algunos miembros de su familia bloquean el proceso de recuperación y empañan las relaciones con el Fondo Monetario Internacional. En Filipinas la destitución del presidente Estrada, acusado de corrupción, ha anclado al país en una inestabilidad que es difícil predecir cómo y cuándo terminará.

\section{El cambio político en el sudeste y este de Asia después de la crisis}

Si después de la crisis las regiones Asia del este y del sudeste son otras, desde el punto de vista económico y financiero, también es cierto que políticamente experimentaron un cambio cualitativo importante. El proceso hacia un ejercicio más transparente, más justo en la actividad política recibió un nuevo impulso, mismo que en algunos países como Tailandia permitió la aprobación de una nueva Constitución, la cual establece reglas más democráticas para las elecciones y para la supervisión del gobierno.

Con excepción de Malasia, dadas las limitaciones que se imponen a la oposición, en los otros cuatro países más afectados por la crisis la oposición relevó del poder a los grupos que no tuvieron la visión para prevenirla: así pasó en Tailandia donde el partido demócrata regresó al gobierno, en Indonesia se acabó con el control dictatorial de 32 años del general Suharto y se abrieron los cauces para la realización de las primeras elecciones libres en el país, que iniciaron el tránsito al cambio democrático; en Corea triunfó la oposición representada por el Partido Congreso Nacional para una Nueva Política, encabezado por Kim Dae Jung, otrora perseguido político de regímenes anteriores; en Filipinas, Joseph Estrada, controvertido ex senador y popular actor, y a pesar de la oposición de la iglesia católica, de los empresarios y del ejército, gana la presidencia.

Así, las regiones del este y del sudeste de Asia de la poscrisis de 1997 son políticamente más democráticas. En varios países los partidos políticos tradicionales, por regla representantes de la alianza de los terratenientesaburguesados, los militares convertidos en empresarios y la burocracia desaparecieron, 0 para sobrevivir establecieron alianzas con las nuevas fuerzas políticas compuestas por una nueva generación de hombres de negocios, producto del boom económico, por la clase media y sectores urbanos radicalizadas por la crisis y por los trabajadores rurales decepcionados por sentirse abandonados a su suerte por los caciques políticos, que ya no les pueden hacer las mismas promesas. Se consolidaron nuevas organizaciones sociales , la población es más participativa, se abre paso la cultura de la defensa de los derechos humanos, hay una mayor transparencia en el ejercicio del poder público, y un incremento en la militancia, nunca vista, de organizaciones no gubernamentales.

A este proceso de cambio, además de la crisis económica, contribuyó el proceso de apertura comercial impuesto por la globalización, uno de cuyos componentes esenciales es la rápida expansión de las nuevas tecnologías de la información, en particular el internet.

En Asia del este y del sudeste, en especial, dado el predominio de una cultura basada en 
las ideas de Confucio que hacen del respeto a la autoridad y a los mayores una condición sine qua non de la conducta social, familiar, política y empresarial, que rechaza el individualismo occidental, la difusión del uso del internet está erosionando ese modo de vida, ese "valor cultural" dirán algunos. Ello lleva implícito un reto demoledor al ancestral control que ejercen los grupos tradicionales (burocracia, militares, las redes de familia en los negocios). Esa rebeldía de la generación de la poscrisis comparte la necesidad del cambio democrático pero desconfía de los actuales políticos, porque no está ya de acuerdo con sus prácticas de padrinazgo, los rechaza por su corrupción.

La generación de la poscrisis rápidamente asimila las nuevas tecnologías de la informática no sólo para sus negocios sino, y para, organizar su vida. Se rompen costumbres, el asiático aprende a no depender más de la empresa o de la familia, empieza a surgir la iniciativa personal y la cultura del riesgo en los negocios.

En la edición especial The New Asia de la revista Newsweek, de julio-septiembre del 2000 se dice que " en la medida en que Asia sale de crisis financiera de fines de 1997, una nueva generación, más segura de sí misma, está floreciendo (blossoming)." Adaptados al este y al oeste dejan de lado la jerarquía practicada por sus padres y optan por técnicas de negocios más transparentes, "No tenemos por qué seguir comprometidos con las formalidades"..nosotros estamos rompiendo con las tradiciones" y lo que más ha difundido ese nuevo espíritu de individualismo es el internet( pp. 9-10). Así en

\section{Cuadro 5}

Porcentaje de población que usa internet, en \%

\begin{tabular}{lcc}
\hline & 1999 & 2005 \\
\hline Singapur & 26.7 & 68.7 \\
Hong Kong & 21 & 57.6 \\
Taiwan & 21.1 & 50.8 \\
Corea del Sur & 8.8 & 27.6 \\
Malasia & 5.5 & 14.5 \\
Tailandia & 1.6 & 5.2 \\
China & 0.4 & 3.9 \\
Filipinas & 0.8 & 3.5 \\
Indonesia & 0.5 & 2.9 \\
\hline
\end{tabular}

Fuente:Newskeek special issue julio-septiembre 2000, p.13 la era cibernética, la vieja Asia paternalista, la de los negocios entre familias, la de las transacciones secretas, se esta acabando.

La crisis económica también contribuyó a dar un nuevo contenido a la ancestral interrelación Asia- Occidente. Ahora se observa en Asia una aceptación generalizada de lo occidental tanto de sus técnicas de negocios como de sus expresiones culturales. En opinión de algunos ello significa que lo occidental se impuso finalmente en Asia, o parafraseando al profesor Walden Bello se afirmaría que ha muerto el "southeast Asia's fast track capitalism" y el capitalismo de estado dirigido, tipo Japón. Yo creo que de lo que somos testigos es de una cohabitación más natural de los valores occidentales y asiáticos.

La interrelación Asia-Occidente (comercial, militar, educativa), gracias a la crisis y a la globalización, se desarrolla sobre la base de nuevos esquemas de cooperación; por ejemplo, en el contexto del Mecanismo de Cooperación Asia Pacífico (APEC), el diálogo político Asia Europa dentro del esquema de ASEM o vía el Foro de Cooperación América Latina-Asia del Este, cuya primera ministerial se desarrolló en Santiago de Chile, los días 29-30 de marzo de 2001, "marca el inicio de un inédito diálogo y cooperación entre ambas regiones para tratar asuntos políticos, culturales, sociales, económicos e internacionales de interés común".

Así, Asia del este y del sudeste de la poscrisis son regiones que se han dado cuenta de que deben avanzar rápidamente, en su integración regional, hacia un mercado común asiático; algunos analistas ya hablan de la necesidad de construir una comunidad asiática. Esa es la alternativa que se percibe en tales regiones para estar en condiciones de competir en el comercio mundial frente a la existencia de otras agrupaciones regionales como la Unión Europea, o el Tratado de Libre de Libre Comercio de América del Norte (NAFTA). Por lo anterior, a pesar de las resistencias que persisten en Asia del este y del sudeste se está definitivamente 
comprometido con la apertura comercial, con la reforma del sistema financiero internacional, con la lucha contra la corrupción, con el tránsito hacia una mayor democracia y la defensa de los derechos humanos.

La nueva realidad es que los dirigentes políticos, los militares y los líderes empresariales de Asia y de Occidente, en la mayoría de los casos, se conocen porque asistieron a la misma Universidad, porque trabajan en alguna filial de la misma empresa $o$ porque asisten a las mismas conferencias internacionales; igualmente en nuestros días, no hay metrópoli importante, asiática u occidental, que no presuma de alguna muestra de inter influencia, sea cultural o gastronómica.

\section{La Asociación de Países del Sudeste (AN SEA) de la poscrisis}

Para presentar una reseña sobre el impacto en los organismos regionales del sudeste asiático debido a la crisis de 1997 considero útil hacer una sucinta introducción de la experiencia de la cooperación regional en dicha zona. Para quienes se interesen en tener una visión más amplia de la evolución reciente de la región, existe una amplia bibliografía que se puede consultar. ${ }^{34}$

Asia del sudeste es la región al sur de China y al este de la India. Su geografía es muy diversa: se extiende a través de tres husos horarios y por su dimensión es comparable a Europa. Exceptuando una parte de Myanmar (Birmania), toda la zona es de clima tropical. Los vientos conocidos como " monzones " son el fenómeno climático que ha influenciado en el modo de vida de los países de la región; el conocimiento de los mismos permitió además a los pueblos del sudeste asiático ser expertos navegantes en el mar de China y el océano Índico.

La región conocida como sudeste asiático es muy diversa también en su cultura, religión e idioma. Las influencias culturales y religiosas que primero recibieron los pueblos de la zona fueron de China y de la India. Para China, esa región fue conocida como "Nan Yan" y de aquel país llegaron las doctrinas del taoismo y del confucionismo, y en la India se le llamó "Svarnadwiya" donde sus monjes misioneros introdujeron el hinduismo y, vía Sri Lanka, llegó el budismo, como lo prueban, a pesar de las inclemencias del tiempo y la destrucción humana, los excelentes templos de Angkor, Borabudur o los múltiples templos de Pagán. A esas herencias culturales siguió la de los comerciantes árabes, quienes llamaban a la zona "Qumr" a la que por muchos años monopolizaron el comercio de las especies y de otros productos vía Europa. Con el contacto con los comerciantes árabes llegó el Islam. A partir del siglo XVI se inició la penetración europea: primero con la presencia portuguesa, vía el océano Índico, luego la de los españoles, vía el Pacífico; después los holandeses, los ingleses y los franceses. La colonización europea dejó su influencia cultural, lingüística y religiosa: catolicismo, protestantismo. Así, en el sudeste asiático se desarrolló uno de los claros ejemplos de sincretismo religioso y cultural.

Los pueblos que hoy integran lo que llamamos sudeste asiático, no se reconocieron por muchos años como parte de una misma región ni se identificaban como herederos de las mismas influencias culturales y religiosas. Por siglos compartieron la región pero vivieron de espaldas unos con otros y, más aún, haciéndose la guerra entre sí, compitiendo por someter a sus vecinos. En razón de esa mutua discordia era frecuente que buscaran protección fuera de la región contra las amenazas de sus vecinos. Ella explica la influencia política de la corte china, a la cual la mayoría de las naciones de la región le pagaban tributo. Es conocido que los gobernantes locales a cambio de protección otorgaron las primeras concesiones comerciales y permitieron la construcción de los centros de acopio y fortalezas militares europeas.

El nombre "sudeste asiático" todavía hacia finales del siglo XIX no se había acuñado. Los europeos que se aventuraron en la búsqueda de las especias llamaban a la región "India Orientalis". El término aparece durante la Segunda Guerra Mundial, cuando los ingleses crean el Comando Militar de Asia del Sudeste. 
Luego, con la formación de la Organización del Tratado del Sudeste de Asia (SEATO, por sus siglas en inglés) alianza militar promovida por los Estados Unidos, la cual por cierto, a pesar de su nombre no fue estrictamente una organización del sudeste asiático, pues la mayoría de sus miembros eran de fuera de la zona. En todo caso fue a partir de esa experiencia que el término sudeste asiático fue generalizado.

Los países que en nuestros días integran el sudeste asiático son Brunei Darussalam, Camboya, Malasia, Myanmar, Laos, Filipinas Indonesia, Singapur, Tailandia y Vietnam; representan más de 500 millones de personas que hablan alrededor de 1000 lenguas, clasificadas en nueve grupos: cínico, para las comunidades de origen chino; tibetano-burman, karen, miao-yao, tai(para Tailandia y Laos); malayo-polinesio mon-khmer, vietnamita y papuan.

Actualmente los habitantes del sudeste asiático practican las tres grandes religiones del mundo: catolicismo, predominante en Filipinas, budismo(therevada) en Myanmar, Laos, Camboya y Tailandia, y el islam sunita en Brunei, Malasia e Indonesia. Por cierto este último es el país con mayor número de musulmanes en el mundo. En Singapur, dado que su población es mayoría de origen chino, predomina el confucionismo, y en Vietnam hay un sincretismo del confucionismo y budismo mahayana. En todos los países hay sectores de la población que practican otras religiones, principalmente el hinduismo (Tailandia, Malasia, Singapur e Indonesia), el islam( en Filipinas, Tailandia), y catolicismo( Laos, Tailandia, Myanmar, Vietnam, Indonesia).

El sudeste asiático es el principal productor de abacá, de más de 85 por ciento del caucho, de 85 por ciento del aceite de palma, de 70 por ciento del estaño, de las dos terceras partes de la copra; es una de las grandes zonas productoras de arroz. Tiene una importante producción de petróleo, considerables depósitos de oro, cobre y de otros minerales preciosos. Además es en nuestros días una regiónque ocupa un importante lugar como exportador de productos electrónicos, textiles, eléctricos, azúcar, frutas y pesquerías.

\section{La creación de la Asociación de Naciones del Sudeste de Asia (AN SEA)}

Como se apunta en párrafos anteriores, la primera agrupación de tipo regional, pero en la cual sólo participaron dos países de la zona, Filipinas y Tailandia, fue la Organización del Tratado del Sudeste de Asia( SEATO), instrumento establecido por Estados Unidos de América y sus aliados para aplicar el Tratado de Defensa Colectiva de Asia del Sudeste, firmado en 1954, y más conocido como Pacto de Manila, cuyo objetivo central fue brindarse ayuda mutua para detener el avance del socialismo en el sudeste de Asia y el Pacífico del sur. Esa organización, cuyas oficinas centrales estuvieron en Bangkok, respondió a las preocupaciones de seguridad que suscitaron los triunfos de las fuerzas comunistas en China, Corea del Norte, Laos y Vietnam.

La SEATO, confirmó la existencia, en el Sudeste Asiático, de tres grupos de países: el pro occidental-capitalista, en torno a la SEATO, el pro comunista y los neutrales, como Indonesia y Camboya. Después de la derrota de Estados Unidos en Vietnam, en 1973, dicha organización se extinguió en 1977.

Los primeros intentos endógenos de crear una asociación de países del sudeste de Asia fue la formación, en 1960, de la organización Maphilindo, que es una amalgama de los nombres de sus fundadores: Malasia, Filipinas e Indonesia, que tuvo corta vida. Luego Malasia, Filipinas y Tailandia crearon la Asociación del Sudeste de Asia( ASA, por sus siglas en inglés), que también duró poco. Sin embargo esas experiencias contribuyeron a dar origen a la primera organización con mayor participación regional, la Asociación de Naciones del Sudeste de Asia(ANSEA) establecida por la Declaración de la ANSEA, también conocida como Declaración de Bangkok, del 8 de agosto de 1967, firmada en la capital tailandesa por cinco países: Malasia, Filipinas, Indonesia, Singapur y 
Tailandia. El grupo de países que integran lo que se conoce como Indochina, Camboya, Laos, y Vietnam, que además en esa época formaban parte del campo socialista, más Birmania (Myanmar ahora) que se se mantenía autoaislada, permanecieron fuera de esa nueva asociación.

En el período comprendido entre el fin de la guerra en Vietnam y la desintegración del campo socialista, la ANSEA se mantuvo prácticamente paralizada por la rivalidad en la zona entre la República Popular China y la ex URSS, expresada en la lucha por el control de Camboya y Laos. Contienda en la que los actores en primer plano fueron el Khmer Rojo, del lado chino, y Vietnam del lado soviético.

Es útil recordar que en vísperas del 36 aniversario de la ASEAN los objetivos centrales que se fijaron sus fundadores fueron en el preámbulo de la Declaración de Bangkok se reconocióla existencia de intereses y problemas comunes, el deseo de promover la cooperación regional dentro del espíritu de igualdad y amistad y así contribibuir a la paz, al progreso y la prosperidad regional.

En los 36 años de existencia la ANSEA ha ido evolucionando lentamente, sus miembros durante la primera década de la organización se acostumbraron a hablarse entre sí, a crear un ambiente de confianza, llamaban a trabajar juntos. Una explicación de esa cautela fue los conflictos en la región, en primer término la guerra de Estados Unidos contra Vietnam y el apoyo que varios países de la ASEAN le brindaron; en segundo lugar las divergencias entre los propios miembros de la Asociación. Por ello, en su primera reunión cumbre, en Bali en 1976, 19 años después de su creación y al término de la guerra en Vietnam, se acordó dar prioridad a la lucha contra la pobreza y fortalecer la identidad regional; para ese fin se aprobó la Declaración ASEAN Concord, misma que se acompaño de un Programa de Acción y de un Tratado de Amistad y Cooperación.

Con la derrota del régimen sanguinario del Khmer Rojo y la formación en Camboya de un gobierno de unidad nacional, con apoyo de la ONU, hechos que casi coinciden con el fin de la Guerra Fría, para la ANSEA se abrió la oportunidad, por primera vez, de convertirse en una verdadera agrupación regional, abriendo sus puertas a los antiguos enemigos ideológicos. ${ }^{35}$

También, como consecuencia del fin de la división del mundo en dos bloques ideológicos, las prioridades de los países como Estados Unidos, Japón y de las ex potencias coloniales europeas se redefinen y pasa a primer término la lucha por el control de los mercados y, en ese contexto, se vuelve prioritario presionar para imponer la economía de mercado, la supresión de las barreras arancelarias y la libre circulación del capital.

Los países del sudeste de Asia con economía capitalista son: Malasia, Filipinas, Indonesia, Singapur y Tailandia y, ricos en mano obra barata y recursos naturales, adquieren una importancia estratégica de primer orden para la expansión de las compañías trasnacionales; primero para las japonesas y después para las de otros países. Así, desde la segunda mitad de la década de 1980 a 1997, las economías de dichos países crecieron a ritmos sorprendentes, lo cual se expresó en una clara elevación del nivel de vida, la consolidación de la clase media, la de la cultura urbana en detrimento del modo vida rural. Ese éxito permitió a todos los observadores de la zona hablar del "milagro del sudeste asiático. ${ }^{36}$

En este contexto, la ANSEA desplegó mayor actividad en la promoción de la cooperación e identidad regional. El ingreso de Vietnam a la ANSEA, en 1995, de Laos y Myanmar en 1997 y finalmente de Camboya en 1999, marcan sin duda un cambio fundamental en esa agrupación. Primero, desde el punto de vista político-ideológico, la organización se volvió pluralista, pues se aceptaba a dos nuevos miembros que siguen auto considerándose socialistas con un férreo régimen militar $\mathrm{y}$, finalmente, que adquirió su carácter genuinamente regional. 


\section{Fuentes}

1 Revista Mexicana de Política Exterior Núm. 57, junio de 1999, "Economical and social survey of Asia and the Pacific" 1998, 1999 y 2000, United Nations Publication; Trade and Development Report 1998, 1999 de la Conferencia sobre Comercio y Desarrollo de la ONU; anuarios de Far Eastern Economic Review, 1998,1999 y 2000; Asian economic crisis: causes, consequences and policy lessons, editado por la Comisión Económica y Social de la ONU para el Asia y el Pacífico ( ESCAP), United Nations, New York, 1999; "La crisis en..." Revista de Comercio Exterior del Banco Nacional de Comercio Exterior

2 Yilmaz Akyuz Causes and sources of the Asian financial crisis, Simposium on Economic and Financial recovery in Asia, UNCTAD X, 17 february 2000, Bangkok, p .6

3 Philip Kotler and Herman Kartajaya, Repositioning Asia, from bubble to sustainable economy. published by John Wiley and Sons Singapore, 2000. p.IX.

4 Anand Panyarachun, ponencia en Eighth Pacific Basin Finance Economics and Accounting Conference, 1o. de junio de 2000, tomado de la versión publicada en el diario The Nation, del 2 de junio del 2000 ..

5 Lee KuanYew, discurso en el Colegio Nacional de Defensa de Tailandia. Tomado de la versión publicada en el diario The Nation, del 23 de junio de 1998.

6 Lee Hsien Loong, entrevista publicada en diario Bangkok Post, 29 de marzo del 2000.

7 Lee Hsien Loong, "Drawing and implementing the lessons of the Asian crisis," The International Herald Tribune, 22 de septiembre del2000.

8 Dr. Azizul Islam, director of Development Research and Policy Analysis Division, Escap. Comentarios en el Simposium sobre la Recuperación Económica y Financiera en Asia,17 de febrero de 2000 Bangkok. UNCTAD X, Bangkok, julio de 2000, p. 49

9 Philip Kotler, ibidem, pp. IX-X.

10 Economic and Social Survey of Asia and the Pacific, 1999, Economic and Social Commission for Asia and the Pacific. United Nations, New York, 1999 p. 118.

11 Ibidem, p. 119.

12 Ver detalles de dichos programas en el estudio de la Economic and Social Comision for Asia and the Pacific, ibidem, pp.126-134.

13 Amy Tong, "Hong Kong property market still idle", en The Hikkei Weekly, 22 de enero del 2001.

14 Walden Bello,Southeast Asia's " fast track" capitalism, The Nation(Bangkok) 4 de diciembre de 1997.

15 Krugman, Paul, "The return of depression economics", pp. 56-74, Foreign Affairs, vol. 78. Núm.1 january, february 1999.

16 "East Asia recovery and beyond", The World Bank, Washington, D.C., may 2000, Pág.1.

17 Ibidem.

18 Ver Forum to debate Asian recovery, en The Nation, 6 de junio del 2000.

19 "New crisis coming, discurso de Alan Greenspan en el Consejo para las Relaciones Internacionales, Nueva
York, 12 de julio del 2000; ver Bangkok Post, 14 de julio de 2000

20 Economic forecasts for Asian industrializing region. Edited by Mitsuru Toida and Daisuke Hiratsuka,Institute of Developing Economies, IdeJetro, Japan, 2000. p. 9

21 Ver al respecto, Karen Richardson, Asian Property Markets Show Signs of Recovery, en The Asian Wall Street Journal, 14/3/2001.

22 Más sobre el impacto de la crisis en el sector inmobiliario en Property Hangover, Far Eastern Economic Review, 7 de diciembre del 2000.

23 Ver DBS Group Holdings, The Asian miracle: back on course... en Euromoney junio del 2000 p. 11.

24 Ver "The Asia week financial 500, the region's largest banks 2000", vol.26,núm.36, september 15, 2000

25 Ibidem, p. 52

26 "Asia Recovery Report 2000", october issue, ADB, Manila, Philippines, p.3

27 ver más al respecto en "Asia Recovery Report 2001", Asian Dt Bank, march 2001, pp. 11-12.

28 Más sobre la experiencia asiática al respecto consultar el capítulo VI del informe Económico y Social de ESCAP 2000.

29 ver Banco Asiático del Desarrollo (ADB por sus siglas en ingles) en su pronóstico para los países más afectados por la crisis en “Asia Recovery Report 2001”, publicado en marzo del 2001; comparte las conclusiones de ESCAP, en cuanto a sus logros, a su recuperación en el 2000 y respecto a los desafíos que enfrentan.

30 Tom Holland, “Asia's New Fissure," Far Eastern Economic Review, june 29 2000, p. 14

31 Michael Richardson in "east Asia, an ineven recovery raices fears," International Herald Tribune, 5 de agosto $\operatorname{del} 2000$.

32 "South-East Asia's problem trio", The Economist, 2 de diciembre del 2000, p. 29

33 Jeffre A. Winters, "The dark side of the tigers", The Asian Wall Street Journal, 12-13 de diciembre de 1997.

34 Entre las obras de carácter general está el libro de John F. Cady, Southeast Asia, its historical development, Mc. Graw -Hill, Nueva Delhi, 1964; Milton Osborne, Southeast Asia an introductory history, sexta edición, Silkworm books, Tailandia, 1995; Niels Mulder, Inside Southeast Asia, 1a. edición, Pepin Press, 1996; Jim Rohwer, Asia Rising, Simon and Schuster N.Y., 1995; John Naisbitt, Megatrends Asia, Nicholas Brealey, Londres, 1995; Michael R.J. Vatikiotis, Political Change in Southeast Asia, Routledge, EUA, 1996; Ian Marsh, Democracy, governance and economic performance: east and southeast Asia, United Nations Press, tokyo, 1999; Mohathir Mohammad, A new deal for Asia, pelanduck publications, Malasia, 1999: Faith Keenan, The aftershock, Review Publishing, Co. Ltd., Honk Kong, 1998.

35 Despúes de los cinco países fundadores: Malasia, Filipinas, Indonesia, Singapur y Tailandia; ingresaron Brunei en 1987; Vietnam en 1995; Laos y Myanmar en 1997 y Camboya formalizaron su admisión en 1999. 


\section{Temas varios del Pacífico}

36 sobre esos cambios en el sudeste asiático ver Jim Rhower, Asia Rising, simon and Schuster, 1995; Frank B. Tripton, the rise of Asia, economics, society and politics in contemporary Asia, MacMillan Press, 1998, en especial capítulo 13; y sobre la idiosincrasia de los pueblos de la región una buena guía es el libro de Niels Mulder, Inside southeast Asia: religion, every day life, cultural change, Pein Press 1996. TI: 